\title{
Effect of laser intensity on the determination of intermolecular electron transfer rate constants-Observation of Marcus inverted region in photoinduced back electron transfer reactions
}

\author{
Yu-Xiang Weng, ${ }^{a), b)}$ Kwok-Chu Chan, Biing-Chiau Tzeng, and Chi-Ming Che ${ }^{\text {a) }}$ \\ Department of Chemistry, The University of Hong Kong, Pokfulam Road, Hong Kong
}

(Received 8 January 1998; accepted 2 July 1998)

\begin{abstract}
The light intensity and concentration dependence of the photoproduct yield are investigated in a monophotonic process. The relationship of the photoproduct yield with the laser intensity and the complex concentration for a monophotonic process is derived under laser flash photolysis. The relationship is confirmed experimentally in a monophotonic process, i.e., triplet-triplet transition for a $\mathrm{Cu}(\mathrm{I})$ complex $\mathrm{Cu}_{6}\left(\mathrm{DMNSN}^{\prime}\right)_{6}\left(\mathrm{DMNSN}^{\prime}=4,6\right.$-dimethylpyrimidine-2-thiolate). At low light intensity, the relationship can be approximated by a linear inverse square root dependence on the light intensity. Based on this equation, a method is proposed to determine the intrinsic back electron transfer rate constant $k_{\mathrm{ET}}^{b}$ in photoinduced intermolecular electron transfer reactions, precluding the effect from the diffusional encounter pairs. The Marcus "inverted region" is observed by using the method in photoinduced back electron transfer reactions of $\left[\mathrm{Au}_{2}(\mathrm{dppm})_{2}\right]\left(\mathrm{ClO}_{4}\right)_{2}$ $(\mathrm{dppm}=$ bis(diphenylphosphino)methane) with a series of substituted pyridinium acceptors.

(C) 1998 American Institute of Physics. [S0021-9606(98)00338-9]
\end{abstract}

\section{INTRODUCTION}

Since the publication of Marcus' electron transfer theory in $1956,{ }^{1,2}$ experimental verification of the "inverted effect" has been the subject of intense study. ${ }^{3-23}$ In earlier works, experiments were performed in intermolecular electron transfer (ET) systems, ${ }^{3-5}$ where ET rate was shown to follow Rehm-Weller behavior, ${ }^{3}$ i.e., in the expected inverted region, the ET rate found was not decreasing with increasing driving force, but remaining pegged at the diffusion limit instead. Experimental evidence for the inverted effect has mainly been obtained with intramolecular ET reaction systems, ${ }^{6-14}$ where the donor and acceptor are covalently linked by intervening spacers. With such a strategy, the diffusion-controlled process can thus be avoided.

Despite the diffusion-controlled process with intermolecular ET reactions, the inverted effect for intermolecular ET reactions has been confirmed in the back ET processes. ${ }^{15-20}$ In liquid media, back ET reactions may not exactly follow a bimolecular kinetics. According to the germinate pair model suggested by Noyes, ${ }^{24,25}$ the germinate recombination should follow the rule of statistical mechanics, and this leads to the deviation of the back ET reaction from conventional kinetics. In 1987, Gould et al. proposed a method to determine the relative back ET rate constant, ${ }^{15-19}$ which measures the absolute quantum yield for radical ions that escape from the germinate pair. The relative constant can be converted to the absolute one with a prerequisite of the knowledge of the dissociation rate constant of the germinate ion pair. The complexity of the kinetics for the germinate pair recombination can be circumvented in this way. In

\footnotetext{
a) Authors to whom correspondence should be addressed.

${ }^{b}$ Present address: Department of Chemistry, Emory University, 1515 Pierce Drive, Atlanta, Georgia 30322. Tel: (404)-727-1419, Fax: (404)-727-6586.
}

fact, most of the evidence for the existence of the inverted region in the intermolecular back ET reactions was achieved by this approach. ${ }^{15-19}$ With a few exceptions, ${ }^{20}$ attempts to observe the inverted effect by direct measurement of the back ET rate have failed. ${ }^{5}$ It has been reported in several cases that the directly observed back ET rate constants would be subjected to the variation of the excitation intensity, ${ }^{26,27}$ which prevents the accurate determination of the back ET rate. The light-intensity effect on the observed rate constant has not yet been studied.

In the present work, we derive equations describing the light intensity and concentration dependence for a monophotonic process. The results are examined in a luminescent hexanuclear $\mathrm{Cu}(\mathrm{I}) \quad$ cluster $\quad \mathrm{Cu}_{6}\left(\mathrm{DMNSN}^{\prime}\right)_{6}$ (DMNSN $^{\prime}=4,6$-dimethylpyrimidine-2-thiolate) which has an octahedral geometry of the metal core. ${ }^{28,29}$ This cluster is suitable for quantitative study of monophotonic and biphotonic processes owing to its stability under photolysis. Though the light-intensity dependence has been studied previously, ${ }^{30-33}$ our theoretical treatment further emphasizes both the light saturation and the inhomogeneous distribution of the species. The relationship between the observed back ET rate constant, intrinsic back ET rate constant, and the initial concentration of the excited-triplet molecule is derived by a model of diffusional encounter pairs. ${ }^{34,35}$ By incorporating the results of light-intensity dependence, a practical method of extracting intrinsic back ET rate constants from the observed values is developed. Application of this method leads to observation of the inverted region in the photoinduced back ET reactions of a dinuclear gold(I) complex $\left[\mathrm{Au}_{2}(\mathrm{dppm})_{2}\right]\left(\mathrm{ClO}_{4}\right)_{2}(\mathrm{dppm}=$ bis (diphenylphosphino) methane) with a series of substituted pyridinium acceptors. 


\section{THEORETICAL CONSIDERATION}

\section{A. Light intensity and concentration dependence in a monophotonic process}

Photoinduced electron transfer is a monophotonic process. Upon the pulsed laser excitation, at low absorbance the light absorption of the solution is described by the BeerLambert law, while at high excitation power, light saturation effect should be considered. During the laser pulse, part of the ground-state molecules are pumped into the lowest excited-singlet state, and the kinetics among the thermally equilibrated lowest excited-singlet and triplet state can be described by Jablonski diagram. ${ }^{36}$

The process of pumping ground-state molecules into their excited states is directly related to the absorption of the ground-state molecules, and the rate constant $k_{p}$ is expressed as $^{37}$

$$
k_{p}(x, t)=\varepsilon I(x, t),
$$

where $I(x, t)$ is the photon flux at a distance $(x)$ inside the sample cuvette from the surface of incidence after $t$ second of excitation, and $\varepsilon$ is the extinction coefficient for the ground-state molecules. Assuming that molecules in the excited states have a negligible contribution to the light absorption, and the Gaussian wave form of the laser pulse is treated as being rectangular with a uniform intensity $I_{0}$, thus $I(x, t)$ can be expressed by the Beer's law

$$
I(x, t)=I(x, 0)=I_{0} e^{-C_{0}(x) \varepsilon x},
$$

where $c_{0}$ is the concentration of the ground-state molecules, and the rate equation for population of the excited-singlet state can be written as

$$
\frac{\partial c_{1}(x, t)}{\partial t}=k_{p} c_{0}(x, t)-\left(k_{r}+k_{\mathrm{ISC}}\right) c_{1}(x, t),
$$

where $c_{1}$ represents the concentration of the lowest excitedsinglet molecules, and $k_{r}$ and $k_{\mathrm{ISC}}$ are the radiative decay and intersystem crossing rate constants, respectively. Compared to the spin-allowed excited-singlet state, population of the spin-forbidden excited-triplet state would be insignificant, and therefore the ground-state depletion due to population of the triplet state can be neglected. Thus the relationship of mass conservation is employed,

$$
c^{0}(x)=c_{0}(x)+c_{l}(x),
$$

where $c^{0}$ is the initial concentration of the compound. Applying the steady-state condition and combining Eqs. (1) to (4) gives

$$
c_{1}(x)=\tau_{f} \varepsilon I_{0}\left(c^{0}-c_{1}(x)\right) e^{-\varepsilon\left(c^{0}-c_{1}(x)\right) x},
$$

where $\tau_{f}$ is the fluorescence lifetime of the compound, and the total number of the excited-singlet molecules along a 1 $\mathrm{cm}$ optical path produced per second at the steady state is

$$
C_{1}=s \int_{0}^{1} c_{1}(x) d x,
$$

where $s$ is the illuminated area. As shown in Appendix A, under the approximation of homogeneous spatial distribution, the above integration can be written as

$$
C_{1} \approx s \tau_{f} I_{0}\left(1-e^{-\varepsilon c^{0} / 1+\tau_{f} \varepsilon I_{0}}\right) .
$$

The triplet-state molecules are generated by intersystem crossing from the singlet state with a quantum efficiency of $\phi_{e m}$, and the total number produced in a single pulse would be

$$
C_{T}=\int_{0}^{\Delta t} k_{\mathrm{ISC}} C_{1} d t=\phi_{e m} s \Delta t I_{0}\left(1-e^{-\varepsilon c^{0} / 1+\tau_{f} \varepsilon I_{0}}\right),
$$

where $\phi_{e m}=k_{\mathrm{ISC}} /\left(k_{r}+k_{\mathrm{ISC}}\right)=k_{\mathrm{ISC}} \tau_{f}, \tau_{f}$ is the lifetime of fluorescence, and $\Delta t$ is the effective pulse width. Equation (8) shows explicitly the concentration of the excited-triplet molecules depends on experimental conditions such as laser intensity and initial concentration.

However, Eq. (8) predicts a monotonic increase of $C_{T}$ with increasing initial concentration $c^{0}$, which inappropriately describes the concentration quenching effect. Therefore, the geometrical factor describing the spatial inhomogeneous distribution should be incorporated. A typical aperture of the detected area is considered to be a rectangular opening of $0.4 \mathrm{~cm}$ in width; therefore, the integration in Eq. (6) should be modified to

$$
C_{1}=s \int_{a}^{b} c_{1}(x) d x,
$$

where $a=0.3$ and $b=0.7$, defining the two margins of the aperture. Thus

$$
\begin{aligned}
C_{T}= & s \phi_{e m} \Delta t I_{0}\left(e^{-\varepsilon c^{0} a / 1+\tau_{f} \varepsilon I_{0} e^{-\varepsilon c^{0} a}}\right. \\
& \left.-e^{-\varepsilon c^{0} b / 1+\tau_{f} \varepsilon I_{0} e^{-\varepsilon c^{0} b}}\right) .
\end{aligned}
$$

Equation (10) is a more general description of the probed triplet-state population (see Appendix B), since the equation includes the light saturation effect, ground-state depletion, and geometrical constraint of the probed area.

\section{B. Light-intensity dependence in bimolecular back ET reaction}

The following scheme has been proposed to describe a photoinduced ET process ${ }^{34,35}$

Scheme 1

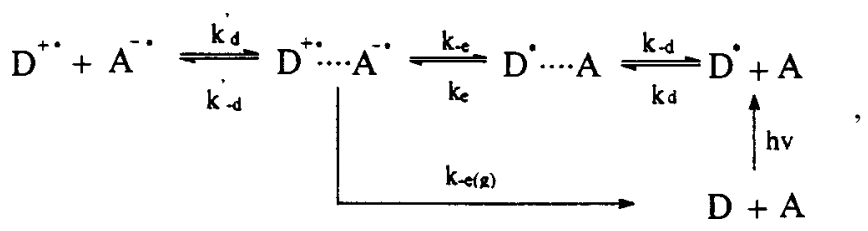

where $D$ and $A$ stand for the donor and acceptor, respectively. In the direct measurement of the back ET rate, the apparent back ET rate constant $k_{\mathrm{obs}}^{b}$ is obtained by a simple second order rate equation. Under the steady-state approximation for the germinate ion pair $D^{+} \cdot \cdots A^{-\bullet}, k_{\mathrm{obs}}^{b}$ becomes (see Appendix C) 


$$
\begin{aligned}
k_{\mathrm{obs}}^{b}= & \frac{k_{-e(g)} k_{d}^{\prime}}{k_{-d}^{\prime}+k_{-e(g)}+k_{-e}}+\frac{k_{-e(g)} k_{e}}{k_{-d}^{\prime}+k_{-e(g)}+k_{-e}} \\
& \times \frac{\left[D^{*} \cdot \bullet A(t)\right]}{\left[D^{+\bullet}(t)\right]\left[A^{-\bullet}(t)\right]},
\end{aligned}
$$

and if $k_{-d} \gg k_{-e(g)}$ and $k_{-e}, k_{\mathrm{obs}}^{b}$ can be written as

$$
k_{\mathrm{obs}}^{b}=\frac{k_{-e(g)} k_{d}^{\prime}}{k_{-d}}+\frac{k_{-e(g)} k_{e}}{k_{-d}} \times \frac{\left[D^{*} \cdot \bullet A(t)\right]}{\left[D^{+\bullet}(t)\right]\left[A^{-\bullet}(t)\right]} .
$$

The first term would be the intrinsic back ET rate constant $\left(k_{\mathrm{ET}}^{b}\right)$, while the second term depends on the concentration of $D^{*} \bullet \bullet A, D^{+\bullet}$, and $A^{-\bullet}$, which are dependent on the excitation light intensity. Numerical computation shows that the relationship between initial concentration of the excited species and the excitation light intensity described by Eq. (8) can be approximated by a simple square root relationship at lower absorbance, i.e.,

$$
\left[D^{*}\right]_{0}=\text { const } \times \sqrt{I_{0}} \text {. }
$$

As shown in Appendix D, under the condition that $\left[D^{+\cdot}\right]_{0} k_{\mathrm{ET}} t \ll 1, k_{\text {obs }}^{b}$ can be expressed as

$$
k_{\mathrm{obs}}^{b}=k_{\mathrm{ET}}^{b}+\text { const } \times\left(1 / \sqrt{I_{0}}\right) .
$$

Equation (14) shows that $k_{\mathrm{obs}}^{b}$ has an inverse square root dependence on the laser intensity, and $k_{\mathrm{ET}}^{b}$ can be obtained by the intercept from linear regression of the experimental data, i.e., $k_{\mathrm{obs}}^{b}$ versus $1 / \sqrt{I_{0}}$.

\section{EXPERIMENT}

The complex $\mathrm{Cu}_{6}\left(\mathrm{DMNSN}^{\prime}\right)_{6}\left(\mathrm{DMNSN}^{\prime}=4,6\right.$-dimethylpyrimidine-2-thiolate) was prepared according to a modified literature method. ${ }^{28,38}$ The elemental analysis of the compound was in good agreement with calculated data. Calculations for $\mathrm{Cu}_{6} \mathrm{H}_{42} \mathrm{~N}_{12} \mathrm{C}_{36} \mathrm{~S}_{6}: \mathrm{C}, 35.65 ; \mathrm{H}, 3.49 ; \mathrm{N}, 13.87$. Found: C, 36.05; H, 3.50; N, 13.80. The preparation, photophysical and -chemical properties of compound $\left[\mathrm{Au}_{2}(\mathrm{dppm})_{2}\right]\left(\mathrm{ClO}_{4}\right)_{2}$ has been reported elsewhere. ${ }^{39,40}$

The ultraviolet/visible (UV/VIS) spectrum was recorded on a Perkin Elmer Lambda 19 spectrophotometer and steadystate emission spectra on a SPEX Fluorolog-2 spectrophotometer. Luminescent lifetime was measured with a Qswitched Quanta Ray DCR-3 Nd:YAG laser [pulse output $355 \mathrm{~nm}$, full width at half maximum (FWHM): $8 \mathrm{~ns}$ ]. The luminescence decay signal was detected by an R928 photomultiplier tube (PMT). Time-resolved absorption signals were measured on a conventional flash photolysis setup with the $355 \mathrm{~nm}$ output of the Nd:YAG laser as the excitation beam. The monitoring light was from a $300 \mathrm{~W}$ continuous wave $(\mathrm{cw})$ tungsten-halogen lamp and was arranged normal to the excitation beam. The transient absorption signals were amplified by a Tektronix AM 502 differential amplifier, then fed to a digitized oscilloscope. Solutions for photophysical measurement were degassed by at least four freeze-pumpthaw cycles.

In measuring the back ET rate, laser power was monitored with an Ophir power meter. The concentration of the quenchers was fixed at $1.0 \times 10^{-3} \mathrm{M}$. $k_{\mathrm{obs}}^{b}$ were extracted

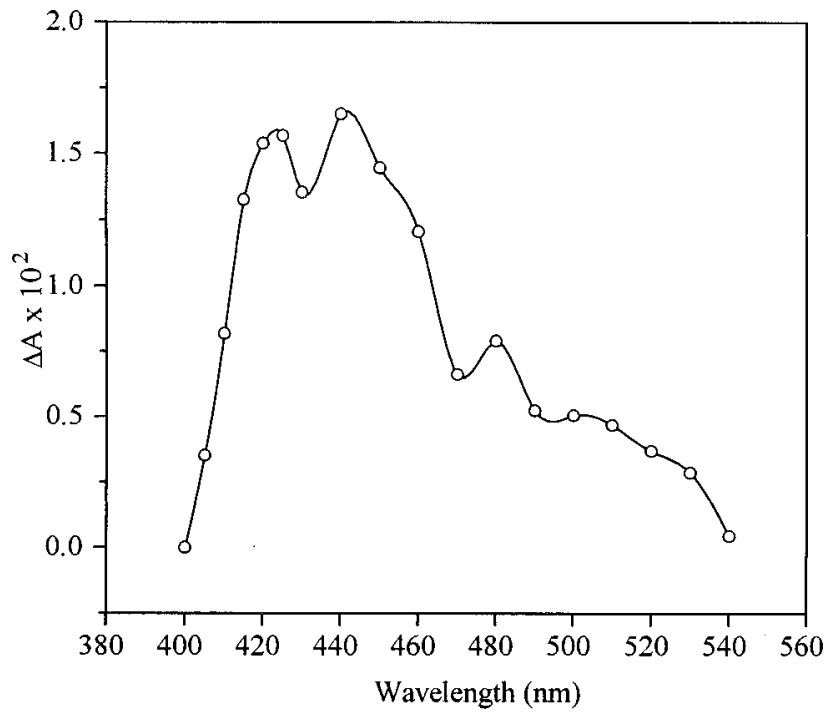

FIG. 1. Triplet-triplet absorption spectrum for a $5.0 \times 10^{-5} \mathrm{M}$ degassed acetonitrile solution of the complex $\mathrm{Cu}_{6}\left(\mathrm{DMNSN}^{\prime}\right)_{6}$ at room temperature acquired at a laser intensity of $1 \mathrm{~mJ} /$ pulse.

from the slope $(m)$ of the absorbance difference $\left(\Delta A^{-1}\right)$ vs. time plots according to $k=\operatorname{lm} \Delta \varepsilon,{ }^{5,20}$ where $l$ is the path length $(1.0 \mathrm{~cm})$ and $\Delta \varepsilon$ is the difference between the sum of the absorption coefficients of the products and those of the reactants at the selected probing wavelength. The transient absorption spectra are dominated by the pyridinal radicals, ${ }^{26}$ and $\Delta \varepsilon$ is approximated as $\varepsilon\left(P y^{\circ}\right)$.

\section{RESULTS AND DISCUSSIONS}

\section{A. Experimental verification of the light intensity and concentration dependence in compound $\mathrm{Cu}_{6}\left(\mathrm{DMNSN}^{\prime}\right)_{6}$}

UV-VIS absorption spectrum shows that the complex has an absorption peak around $285 \mathrm{~nm} \quad\left(\varepsilon_{\max }=2.9\right.$ $\left.\times 10^{4} \mathrm{~mol}^{-1} \mathrm{dm}^{3} \mathrm{~cm}^{-1}\right)$. Upon UV light excitation, the complex shows an emission peak at $713 \mathrm{~nm}$ having a lifetime of 10.0(5) $\mu \mathrm{s}$, and a quantum yield of 0.067. Luminescence quantum yield measurement followed the reported procedure with quinine sulfate in $1.0 \mathrm{~N}$ sulfuric acid as the reference excited at $355 \mathrm{~nm}^{41}$ Figure 1 depicts the triplettriplet absorption spectrum of the complex. The observed absorption decay after flashing the solution with a $355 \mathrm{~nm}$ laser pulse was monoexponential having a lifetime of $9.2 \mu \mathrm{s}$, comparable to the luminescence lifetime. This justifies the assignment of the triplet-triplet absorption spectrum. However, the triplet-triplet absorption did not decay to the background, but exhibited a long-lived residual component with a half-decay time of $\sim 14 \mathrm{~ms}$. The absorbance difference spectrum due to this long-lived component is given in Fig. 2. Figure 3(a) plots the absorbance difference due to triplettriplet transition measured at $420 \mathrm{~nm}$ against the exciting laser intensity at different concentrations. The lines drawn through the experimental points were calculated from Eq. (15), an equivalent of Eq. (8),

$$
\Delta A=N I_{0}\left(1-e^{-\varepsilon c^{0} / 1+\tau_{f} \varepsilon I_{0}}\right) .
$$




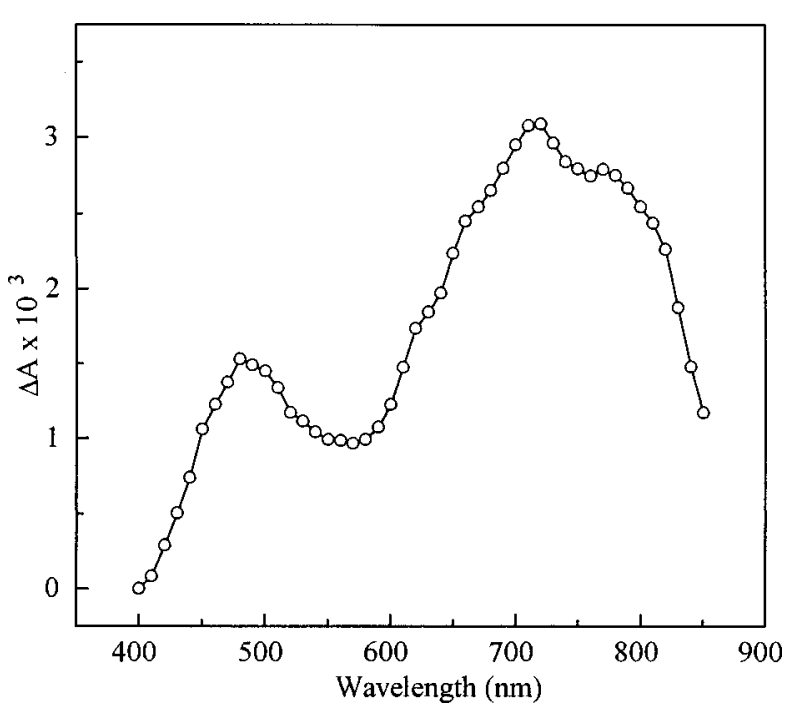

FIG. 2. Absorbance difference spectrum for the photoionized product generated by flashing a $5.0 \times 10^{-5} \mathrm{M}$ degassed acetonitrile solution of the complex $\mathrm{Cu}_{6}\left(\mathrm{DMNSN}^{\prime}\right)_{6}$ at room temperature at a laser intensity of $8 \mathrm{~mJ} / \mathrm{pulse}$, acquired at $500 \mu \mathrm{s}$ after the laser flash.

Here $c^{0}$ is the concentration of the solution, $\varepsilon$ is the extinction coefficient at $355 \mathrm{~nm}$ measured to be 1.1 $\times 10^{4} \mathrm{~mol}^{-1} \mathrm{dm}^{3} \mathrm{~cm}^{-1}, I_{0}$ is the laser intensity, while $N$ and $\tau_{f}$ were treated as fitting parameters. Figure $3(\mathrm{~b})$ describes the fitting of the corresponding experimental data by the linear relationship of inverse square root light-intensity dependence.

Upon increasing the complex concentration, the emission intensity increased at first, then became saturated and eventually dropped off [Fig. 4(a)]. However, the emission lifetime was almost invariant to the concentration change, which indicates that the concentration quenching is due to the spatial inhomogeneity rather than self-quenching effect. ${ }^{42}$ Figure 4 plots the yield of the triplet-state molecule characterized by (a) peak emission intensity; (b) absorbance difference of triplet-triplet transition measured at $710 \mathrm{~nm}$ against the concentration. The solid lines are the calculated curves based on Eq. (10).

As shown in Fig. 2, the absorbance difference spectrum of the residual component is different from that of the triplet-triplet absorption, suggesting the former can not be derived from the monophotonic process. In literature there were reports on organic and inorganic compounds which exhibited long-lived decay components originating from the biphotonic ionization products upon laser flash photolysis. ${ }^{43,44}$ Therefore, we suggest that the residual absorption is due to the biphotonic ionization of the compound $\mathrm{Cu}_{6}\left(\mathrm{DMNSN}^{\prime}\right)_{6}$. When a degassed solution of the compound $\left(5.0 \times 10^{-5} \mathrm{M}\right)$ was reexposed to the dioxygen, the relatively weak residual component disappeared completely, while the phosphorescence lifetime decreased from 10.1 to $1.2 \mu \mathrm{s}$. These imply that the photoionization occurs at the excitedtriplet state rather than the excited-singlet state; therefore, a consecutive biphotonic process ${ }^{30}$ can be suggested for the photoionization of the compound.

As shown in Fig. 3(a), the photoproduct yield in a monophotonic process clearly reveals a saturation effect as laser

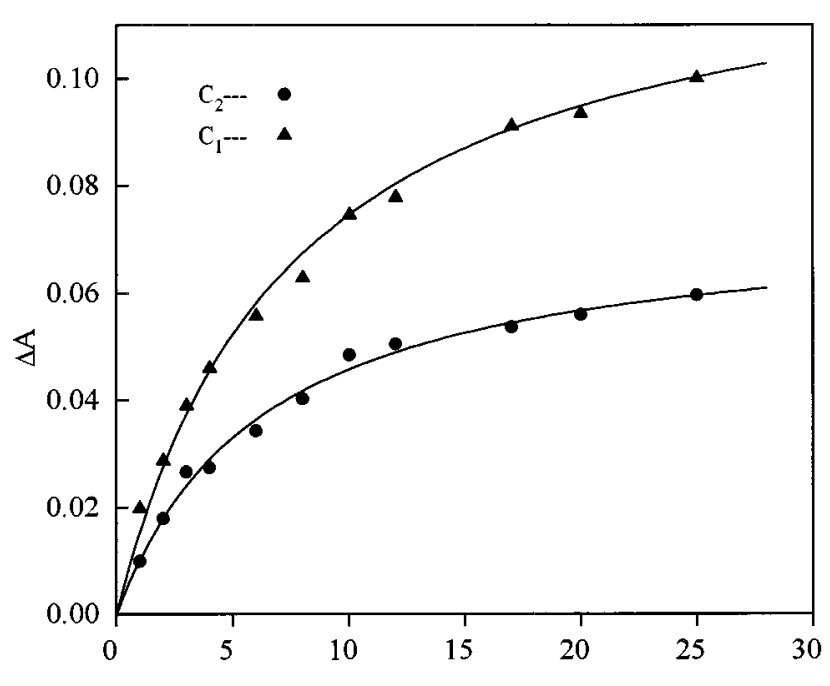

(a)

Laser intensity ( $\mathrm{mJ} /$ pulse)

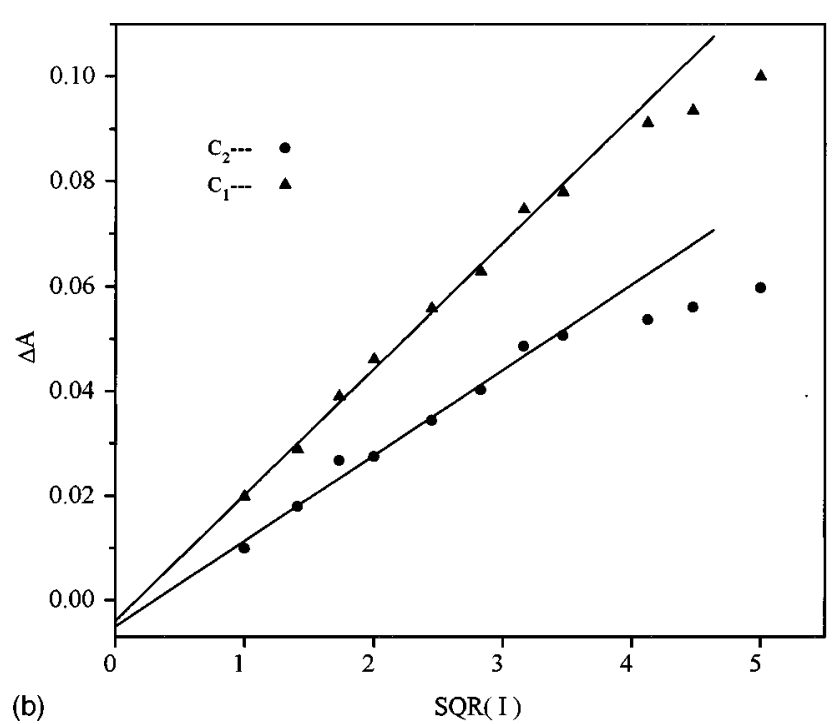

FIG. 3. (a) Plots of the absorbance difference due to triplet-triplet absorption of the complex $\mathrm{Cu}_{6}\left(\mathrm{DMNSN}^{\prime}\right)_{6}$ versus laser intensity at different concentrations. $C_{1}=1.0 \times 10^{-4} \mathrm{M} ; C_{2}=5.0 \times 10^{-5} \mathrm{M}$. The solid lines are calculated curves by Eq. (15) for $N_{1}=2.58 \times 10^{-2}, \tau_{f 1}=2.05 \times 10^{-5}$, and $N_{2}$ $=2.79 \times 10^{-2}, \tau_{f 2}=1.87 \times 10^{-5}$. (b) Linear regression by the square root dependence on the laser intensity in the range of $1-12 \mathrm{~mJ} / \mathrm{pulse}$.

intensity increases. The solid line is the calculated curve by Eq. (15). Figure 3(b) shows that the inverse square root relationship well describes the light-intensity dependence at low laser power range $(1-12 \mathrm{~mJ} /$ pulse), providing experimental evidence for Eq. (13). Similar light-intensity dependence described by Eq. (15) also was observed in the biphotonic process, which shows that the quadratic intensity dependence is not a premise for a biphotonic process. This is consistent with the theoretical prediction ${ }^{30}$ and the recent experimental observation. ${ }^{33}$

In Fig. 4, the concentration quenching effect is attributed to arising from the inhomogeneous distribution of the excited-triplet molecules, which can be rationalized by Eq. (10) setting $N=s \phi_{e m} \Delta t I_{0}, k=\tau_{f} I_{0}$, and $\varepsilon$ as the fitting parameters. Consequently, our results suggest that the inhomogeneous distribution of the excited species caused by nonlinear light absorption can severely affect the measured 

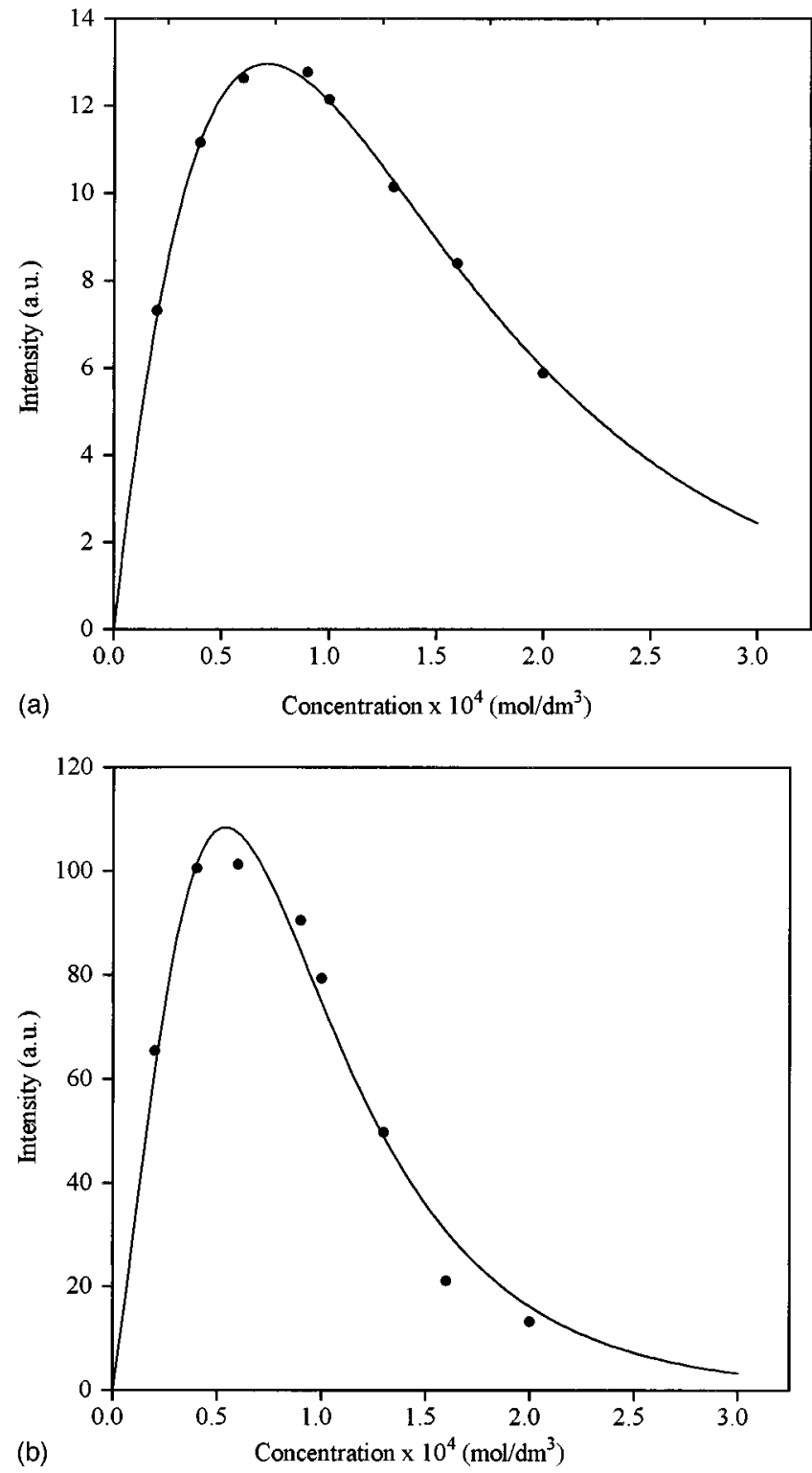

FIG. 4. Concentration dependence of the excited-triplet state for the complex $\mathrm{Cu}_{6}\left(\mathrm{DMNSN}^{\prime}\right)_{6}$ : (a) peak emission intensity; (b) intensity of triplettriplet absorption at a fixed laser intensity of $15 \mathrm{~mJ} /$ pulse. The solid lines through experimental data are calculated by Eq. (10). The fitting parameters are: (a) $\quad N=41 \pm 0.4, \quad k=4.6 \pm 1.4 \times 10^{-6}, \quad$ and $\quad \varepsilon=3.14 \pm 0.04 \times 10^{4}$ $\mathrm{mol}^{-1} \mathrm{dm}^{3} \mathrm{~cm}^{-1}$ (measured $\varepsilon=1.1 \times 10^{4} \mathrm{~mol}^{-1} \mathrm{dm}^{3} \mathrm{~cm}^{-1}$ ); (b) $N=287$ $\pm 16, k=2.0 \pm 0.8 \times 10^{-5}$, and $\varepsilon=5.0 \pm 0.3 \times 10^{4} \mathrm{~mol}^{-1} \mathrm{dm}^{3} \mathrm{~cm}^{-1}$.

luminescence intensity. The commonly employed method for the determination of emission quantum yield ${ }^{41}$ is based on the measurement of emission intensity of the compound compared to that of the reference sample, and the factor of inhomogeneous distribution should be carefully avoided.

\section{B. Experimental verification of the proposed method}

The validity of the proposed method is examined in the photoinduced back ET reaction of $\left[\mathrm{Au}_{2}(\mathrm{dppm})_{2}\right]\left(\mathrm{ClO}_{4}\right)_{2}$ with substituted pyridinium salts. The spectroscopic properties and redox chemistry of the phosphorescent excited state of $\left[\mathrm{Au}_{2}(\mathrm{dppm})_{2}\right]^{2+}$ (here abbreviated as $\mathrm{Au}_{2}^{2+*}$ ) have been reported elsewhere. ${ }^{26}$ The excited-state reduction potential of

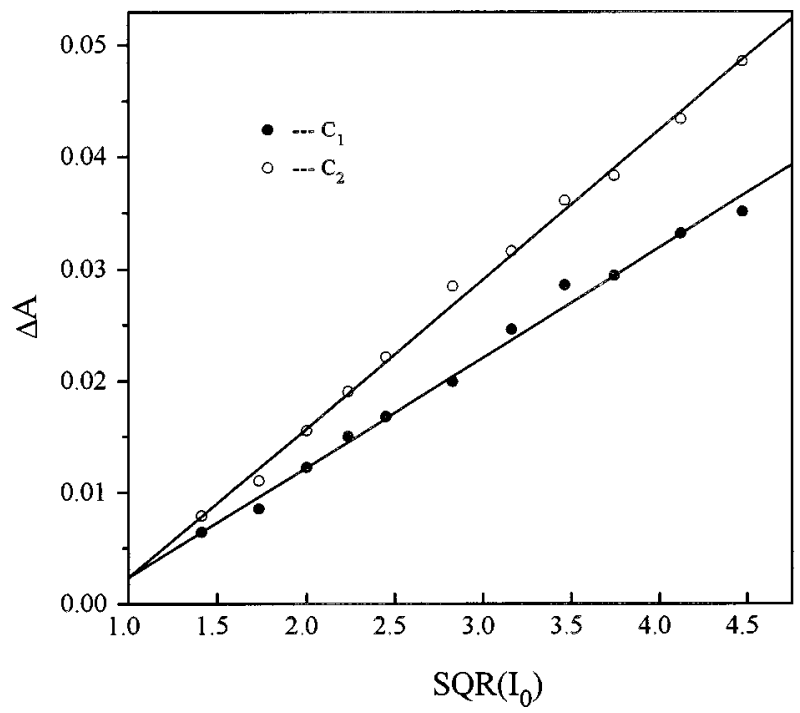

FIG. 5. Plot of absorbance difference of $\mathrm{MV}^{+}{ }^{\bullet}$ recorded $30 \mu$ s after flashing the sample against $1 / \sqrt{I_{0}}$ at a different concentration of $C_{1}\left(\left[\mathrm{Au}_{2}(\mathrm{dppm})_{2}\right]^{2+}\right)=1 \times 10^{-4} \mathrm{M}, \quad C_{2}\left(\left[\mathrm{Au}_{2}(\mathrm{dppm})_{2}\right]^{2+}\right)=2.0 \times 10^{-4} \mathrm{M}$ for the back ET reaction of $\mathrm{Au}_{2}^{3+}$ with $\mathrm{MV}^{++}$. The solid line is the linear fitting curve.

the $\mathrm{Au}_{2}^{3+} / \mathrm{Au}_{2}^{2+*}$ couple was determined to be $-1.6(1) \mathrm{V}$ vs standard saturated calomel electrode (SSCE) by quenching studies, and the 0-0 energy of the excited state $\left(E_{0-0}\right)$ estimated from the emission data is $2.6(1) \mathrm{eV}{ }^{26}$ Thus $E_{1 / 2}$ for the $\left[\mathrm{Au}_{2}(\mathrm{dppm})_{2}\right]^{3+/ 2+}$ couple is estimated to be $1.0(1) \mathrm{V}$. The back ET reactions of $\left[\mathrm{Au}_{2}(\mathrm{dppm})_{2}\right]^{3+}$ with a number of pyridinal radicals were studied, and the reaction schemes are shown as

$$
\begin{aligned}
& \mathrm{Au}_{2}(\mathrm{dppm})_{2}^{2+*}+\mathrm{Py}^{+} \stackrel{k_{q}}{\longrightarrow} \mathrm{Au}_{2}(\mathrm{dppm})_{2}^{3+}+\mathrm{Py} \cdot, \\
& \mathrm{Au}_{2}(\mathrm{dppm})_{2}^{3+}+\mathrm{Py} \cdot \stackrel{k_{\mathrm{obs}}^{b}}{\longrightarrow} \mathrm{Au}_{2}(\mathrm{dppm})_{2}^{2+}+\mathrm{Py}^{+} .
\end{aligned}
$$

The $k_{\mathrm{obs}}^{b}$ of reaction (2) were measured by monitoring the decay of the absorption of the pyridinal radicals. In each case, the decay can be fitted by a second order decay kinetics, but the observed rate constants are dependent on the excitation light intensity and the concentration of $\left[\mathrm{Au}_{2}(\mathrm{dppm})_{2}\right]^{2+}$. If $k_{\mathrm{obs}}^{b}$ has a linear relationship with the inverse square root of the excitation intensity, as predicted by Eq. (14), the $k_{\mathrm{ET}}^{b}$ can be extracted by the proposed method. We begin this by examining the validity of the following conditions necessary to Eq. (14) in a real reaction system.

\section{Determination of the laser-intensity range}

The laser-intensity dependence of the initial concentration of the radical ion is studied in the photoinduced ET reaction of $\left[\mathrm{Au}_{2}(\mathrm{dppm})_{2}\right]^{2+}$ with $N, N^{\prime}$-dimethyl-4, $4^{\prime}$-bipyridinium cation $\left(\mathrm{MV}^{2+}\right)$. The initial concentration of the radical ion $\left(\mathrm{MV}^{+\bullet}\right)$ against the laser intensity can be rationalized by Eq. (15) taking $\varepsilon_{355 \mathrm{~nm}}$ to be $500 \mathrm{~mol}^{-1} \mathrm{dm}^{3} \mathrm{~cm}^{-1} \cdot{ }^{26}$ Figure 5 describes the measured initial concentration $\mathrm{MV}^{+\bullet}$ fitted by the linear relationship of inverse square root light-intensity dependence. The results 


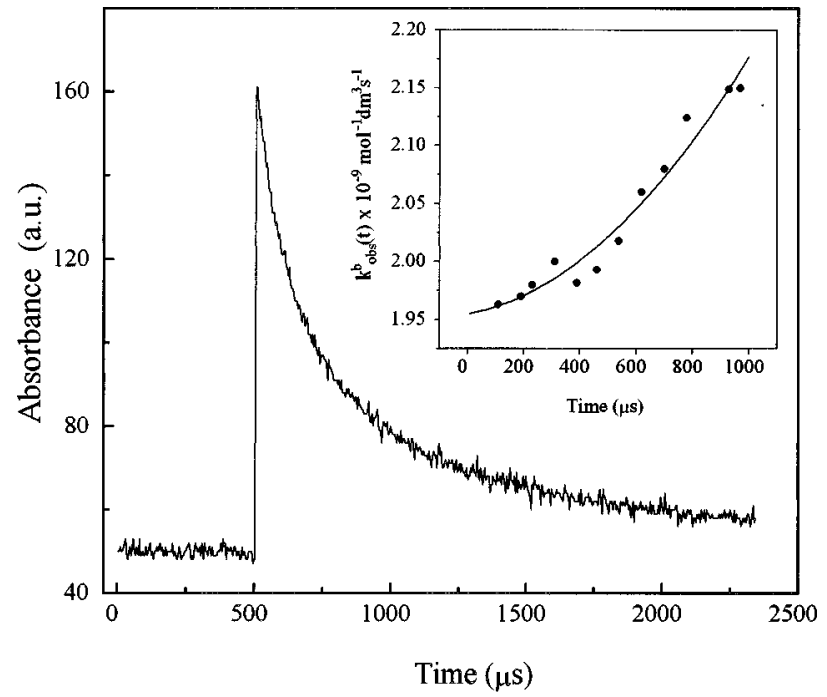

FIG. 6. Typical kinetic trace acquired for the back ET reaction of $\mathrm{Au}^{3+}$ with $\mathrm{MV}^{+*}$, probed at $607 \mathrm{~nm} . \quad C\left(\left[\mathrm{Au}_{2}(\mathrm{dppm})_{2}\right]^{2+}\right)=2.0 \times 10^{-4} \mathrm{M}$; $\mathrm{C}\left(\mathrm{MV}^{+2}\right)=1.0 \times 10^{-3} \mathrm{M}$, at a temperature of $22 \pm 2{ }^{\circ} \mathrm{C}$ and laser intensity of $10 \mathrm{~mJ} /$ pulse. Graphic inset: plot of $k_{\mathrm{obs}}^{b}(t)$ as a function of time; the solid line is calculated by a second order polynomial equation $k_{\mathrm{obs}}^{b}(t)=1.95$ $\times 10^{9}+4.42 \times 10^{5} t+1.78 \times 10^{2} t^{2}$.

reveal that the square root relationship holds as an appropriate approximation for Eq. (8) in the experimental laserintensity range.

\section{Experimental examination for the validity of the condition $\left[D^{+\cdot}\right]_{0} k_{E T} t \ll 1$}

Under the steady-state approximation, at the fixed laser intensity (hence the initial concentration is given), if no approximation is made for the term $\left(1+\left[D^{+\bullet}\right]_{0} k_{\mathrm{ET}} t\right)$, Eq. (14) would be expressed as (Appendix D),

$$
k_{\mathrm{obs}}^{b}(t)=k_{\mathrm{ET}}^{b}+\operatorname{const} \times\left(1+\left[D^{+\cdot}\right]_{0} k_{\mathrm{ET}}^{b} t\right)^{2},
$$

which indicates that the observed bimolecular rate constant is a function of time, i.e., a second order polynomial relationship. Figure 6 presents a typical absorbance decay trace for $\mathrm{MV}^{+\bullet}$ acquired at $10 \mathrm{~mJ} / \mathrm{pulse}$, which can be fitted by a second order kinetics. However, the observed rate constant is varied to the different time range ( 0 to $t$ ) selected for kinetic analysis, showing an increasing tendency with time. The obtained $k_{\mathrm{obs}}^{b}$ versus time can be rationalized by a second order polynomial relation as shown in the graphic inset. The result reveals that in the selected dynamical range, the contribution from the term $\left[D^{+\bullet}\right]_{0} k_{\mathrm{ET}} t$ accounts for only $10 \%$ of the increase in $k_{\mathrm{obs}}^{b}$. Therefore the condition $\left[D^{+\bullet}\right]_{0} k_{\mathrm{ET}}^{b} t \ll 1$ can be satisfied within the dynamical range appropriate for the kinetic fitting. As a result, Eq. (14) can be used to predict the laser-intensity dependence of the $k_{\mathrm{obs}}^{b}$. Figure 7 plots the $k_{\mathrm{obs}}^{b}$ against the laser intensity $\left(1 / \sqrt{I}_{0}\right)$ at two different concentrations of the complex, which shows a good linear relation between the $k_{\mathrm{obs}}^{b}$ and $1 / \sqrt{I}_{0}$. In addition, the concentration effect can also be excluded by using Eq. (14). As shown in Fig. 7, the intercepts for the two different concentrations are consistent within experimental error.

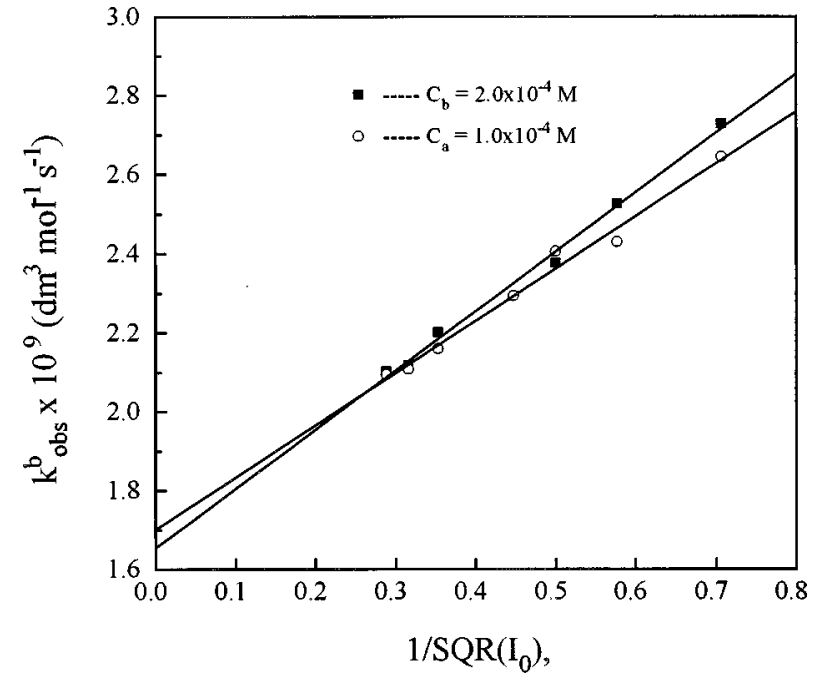

FIG. 7. Plot of $k_{\mathrm{obs}}^{b}$ for the back ET reaction of $\mathrm{Au}_{2}^{3+}$ with $\mathrm{MV}^{+\cdot}$ against $1 / \sqrt{I_{0}}$ at different $\mathrm{Au}_{2}^{2+}$ concentrations, $C_{1}\left(\left[\mathrm{Au}_{2}(\mathrm{dppm})_{2}\right]^{2+}\right)=1.0$ $\times 10^{-4} \mathrm{M}, C_{2}\left(\left[\mathrm{Au}_{2}(\mathrm{dppm})_{2}\right]^{2+}\right)=2.0 \times 10^{-4} \mathrm{M}$.

\section{Observing Marcus inverted region}

Equation (14) gives a general method to determine the bimolecular back ET rate constants. As an example, it is applied to the observation of the Marcus inverted region in the back ET reactions.

The same procedure for the determination of the $k_{\mathrm{ET}}^{b}$ in the back ET reactions of $\mathrm{Au}_{2}^{3+}$ with $\mathrm{MV}^{+\bullet}$ has been applied to the reactions of $\mathrm{Au}_{2}^{3+}$ with a number of other pyridinal radicals, and the measured $k_{\mathrm{ET}}^{b}$ and $k_{q}$ values are listed in Table I. Here $k_{q}$ refer to the quenching rate constants of $\mathrm{Au}_{2}^{2+*}$ by the pyridinium acceptors [Eq. (R1)], which were determined by the Stern-Volmer quenching. ${ }^{26}$ The measured ET rate constants are plotted against the driving force in Fig. 8 , and the data clearly illustrate the existence of an inverted region. The curve in Fig. 8 is calculated with $\lambda_{v}=0.21, \lambda_{s}$ $=0.80 \mathrm{eV}$, and $\omega=1500 \mathrm{~cm}^{-1}$ based on Eq. (17), which treats solvent reorganization classically and includes one quantum-mechanical coordinate for inner-sphere reorganization, ${ }^{7,8,45,46}$

$$
\begin{array}{r}
k_{e t}=\left(4 \pi^{3} / h^{2} \lambda_{s} k_{b} T\right)^{1 / 2}|V|^{2} \sum_{w=0}^{\infty}\left(e^{-S} S^{w} / w !\right) \\
\times \exp \left\{-\left[\left(\lambda_{s}+\Delta G+w h v\right)^{2} / 4 \lambda_{s} k_{b} T\right]\right\}, \\
s=\lambda_{v} / h \nu .
\end{array}
$$

It is apparent that the curve can satisfactorily account for the driving force dependence of $k_{\mathrm{ET}}^{b}$ and $k_{q}$, while the diffusion-limit $k_{q}$ values follow Rehm-Weller behavior. The total reorganization energy is estimated to be $1.01 \mathrm{eV}$, and both the inner sphere $(0.21 \mathrm{eV})$ and outer sphere $(0.80 \mathrm{eV})$ reorganization energy are comparable to those in the photoinduced intramolecular ET reactions of the iridium dimer complex in acetonitrile. ${ }^{13}$ Like most organic intramolecular ET reactions, ${ }^{6,8}$ the $\omega$ value of $1500 \mathrm{~cm}^{-1}$ is attributed to the $\mathrm{C}-\mathrm{C}$ skeletal vibration mode of the pyridinium moiety. This suggests that the photoinduced ET reactions between $\mathrm{Au}_{2}^{2+}$ and the substituted alkyl pyridinium ions proceed via a through-bond path. ${ }^{47}$ 
TABLE I. The $k_{q}$ and $k_{\mathrm{ET}}^{b}$ values for the photoinduced ET reactions between $\left[\mathrm{Au}_{2}(\mathrm{dppm})_{2}\right]^{2+}$ and various pyridinium acceptors in degassed acetonitrile at $22 \pm 2{ }^{\circ} \mathrm{C}$.

\begin{tabular}{lccc}
\hline \hline \multicolumn{1}{c}{ Quenchers\% } & $\begin{array}{c}E\left(A^{+/ 0}\right) \\
(\mathrm{V} \text { vs SSCE })\end{array}$ & $\begin{array}{c}k_{\mathrm{ET}} / \mathrm{dm}^{3} \\
\mathrm{~mol}^{-1} \mathrm{~s}^{-1}\end{array}$ & $\begin{array}{c}k_{\mathrm{ET}}^{b} / \mathrm{dm}^{3} \\
\mathrm{~mol}^{-1} \mathrm{~s}^{-1}\end{array}$ \\
\hline$N, N^{\prime}$-Dibenzyl-4,4'-bipyridinium & -0.35 & & $2.4 \pm 0.1 \times 10^{9}$ \\
$N, N^{\prime}$-Dimethyl-4,4'-bipyridinium & -0.45 & $6.1 \times 10^{9 \mathrm{a}}$ & $1.7 \pm 0.1 \times 10^{9}$ \\
4-Cyano- $N$-methylpyridinium & -0.67 & $5.6 \times 10^{9}$ & \\
4-methoxycarbonyl- $N$ - & -0.78 & $4.5 \times 10^{9}$ & $8.0+12 \times 10^{7 \mathrm{~b}}$ \\
methylpyridinium $_{\text {4-Amido- } N \text {-methylpyridinium }}^{\mathrm{c}}$ & & & \\
4-Amido- $N$-ethylpyridinium & -0.88 & & \\
3-Amido- $N$-ethylpyridinium & -0.93 & $2.9 \times 10^{9}$ & \\
$N$-Ethylpyridinium & -1.14 & $9.5 \times 10^{8}$ & \\
4-Methyl- $N$-methylpyridinium & -1.36 & $1.4 \times 10^{8} \mathrm{a}$ & \\
2,6-Dimethyl- $N$-methylpyridinium & -1.49 & $9.6 \times 10^{6}$ & \\
\hline \hline
\end{tabular}

${ }^{\mathrm{a}} k_{q}$ have been redetermined for ensuring previous measurements; within the experimental error, the data are reproducible. ${ }^{\%}$ : The reduction potentials are cited from Ref. 20, unless it is specified otherwise. The $\varepsilon$ are as follows: $\varepsilon_{607 \mathrm{~nm}}=1.39 \times 10^{4} \mathrm{dm}^{3} \mathrm{~mol}^{-1} \mathrm{~cm}^{-1}$ for $N, N^{\prime}$-dimethyl-4,4'-bipyridinal (Ref. 48 ); $\varepsilon_{400 \mathrm{~nm}}=6.9 \times 10^{3}$ $\mathrm{dm}^{3} \mathrm{~mol}^{-1} \mathrm{~cm}^{-1}$ for 4-methoxycarbonyl- $N$-methylpyridinal (Ref. 49); $\varepsilon_{600 \mathrm{~nm}}=1.6 \times 10^{4} \mathrm{dm}^{3} \mathrm{~mol}^{-1} \mathrm{~cm}^{-1}$ for $N, N^{\prime}$-dibenzyl-4,4'-bipyridinal (Ref. 50); $\varepsilon_{404 \mathrm{~nm}}=7.1 \times 10^{3} \mathrm{dm}^{3} \mathrm{~mol}^{-1} \mathrm{~cm}^{-1}$ for 4-Amido- $N$-methylpyridinal radicals (Ref. 51).

${ }^{\mathrm{b}}$ For a slow recombination rate, the interception is close to the limit of the method, thus only the upper limit could be determined. The error is determined from linear regression.

${ }^{\mathrm{c}}$ Reference 51 .

\section{v. CONCLUSION}

Light-intensity and concentration dependence in flash photolysis measurements were studied theoretically and experimentally. Light saturation effects were observed in both monophotonic and biphotonic processes, which suggests that a biphotonic process can not be simply judged by a quadratic intensity dependence. Concentration quenching can arise from the inhomogeneous distribution of the excited species.

Theoretical treatment based on the diffusional encounter pair model provides a practical method for the determination of the intrinsic back ET rate constant. By systematically tun-

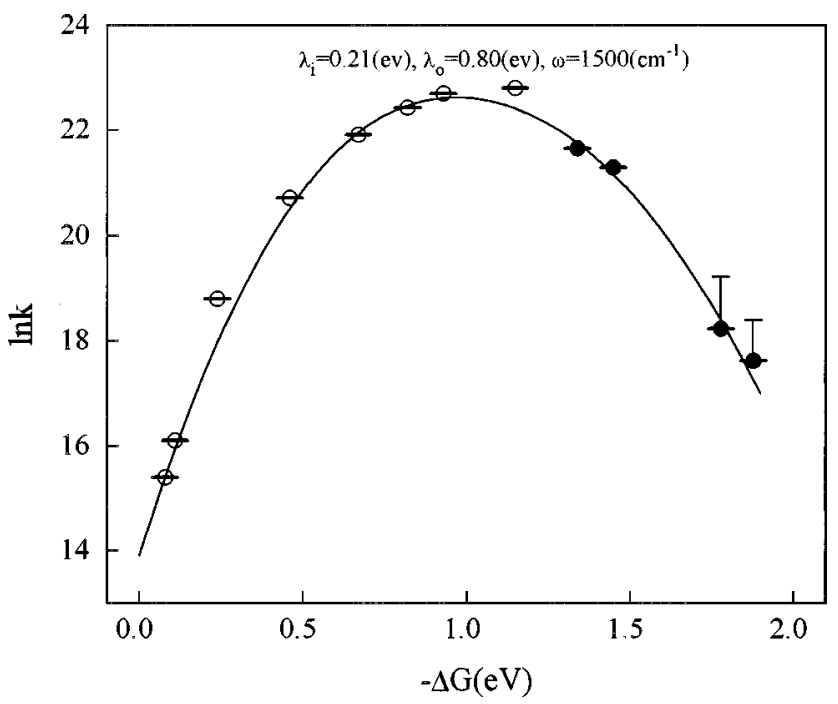

FIG. 8. Driving-force dependence of the electron transfer rates for the photoinduced reactions between $\mathrm{Au}_{2}^{2+}$ and various $N$-alkyl-pyridinium quenchers in acetonitrile solution at room temperature $\left(22 \pm 2{ }^{\circ} \mathrm{C}\right)$ : photoinduced ET reactions $(\bigcirc)$; thermal recombination reactions $(\bullet)$. All the rate constants were corrected for diffusion assuming the limiting rate constant of $2.5 \times 10^{10} \mathrm{M}^{-1} \mathrm{~s}^{-1}$. ing the laser intensity, $k_{\mathrm{ET}}^{b}$ can be obtained from the intercept of the linear extrapolation of $k_{\mathrm{obs}}^{b}$ versus the inverse square root of the laser intensity. As a result, we have developed a method which can determine the intrinsic bimolecular back ET rate constants by using flash photolysis. One application of this method is to observe the Marcus inverted region in the intermolecular back ET reactions. However, the method is based on the linear extrapolation; thus the dynamical range defined as the ratio of the maximum of $k_{\mathrm{obs}}^{b}$ over $k_{\mathrm{ET}}^{b}$ is limited, and a range of $10^{2}$ in magnitude is assumed. Nevertheless, we believe that the method is also applicable to other bimolecular reactions induced by flash photolysis, where the observed rate constant may be subjected to variations in laser intensity.

\section{ACKNOWLEDGMENTS}

We acknowledge support from The University of Hong Kong, the Croucher Foundation, and the Hong Kong Research Grants Council. Y.-X. Weng acknowledges the post doctoral fellowship from the University of Hong Kong.

\section{APPENDIX A}

\section{Derivation of Equation (7)}

The explicit expression for Eq. (6) has the following form:

$$
C_{1}=s \int_{0}^{1} \tau_{f} \varepsilon I_{0}\left(c^{0}-c_{1}(x)\right) e^{-\varepsilon\left(c^{0}-c_{1}(x)\right) x} d x
$$

which is equivalent to 


$$
\begin{aligned}
C_{1}= & -s \int_{0}^{1} \tau_{f} I_{0} e^{-\varepsilon\left(c^{0}-c_{1}(x)\right) x} d\left[-\varepsilon\left(c^{0}-c_{1}(x)\right) x\right] \\
& +s \int_{0}^{1} \tau_{f} I_{0} e^{-\varepsilon\left(c^{0}-c_{1}(x)\right) x} x d\left[-\varepsilon\left(c^{0}-c_{1}(x)\right)\right] .
\end{aligned}
$$

For a system of homogeneous distribution, the second term in the right-hand side of the equation falls to zero naturally, while for an inhomogeneous one, if the deviation from the homogeneous distribution is not significant, the second term can be neglected. Under this approximation and after integration, Eq. (A2) becomes

$$
C_{1}=-s \tau_{f} I_{0} e^{-\left.\varepsilon\left(c^{0}-c_{1}(x)\right) x\right|_{0} ^{1}} .
$$

Thus

$$
C_{1}=s \tau_{f} I_{0}\left(1-e^{-\varepsilon\left(c^{0}-c_{1}(1)\right)}\right) .
$$

Assuming $I(x) \approx I(0) \approx I_{0}$, Eq. (5) could be simplified to

$$
c_{1}(0)=c_{1}(1)=c_{1}(x)=\tau_{f} \varepsilon I_{0}\left(c^{0}-c_{1}(x)\right)
$$

and

$$
c_{1}(1)=\frac{\tau_{f} \varepsilon c^{0} I_{0}}{1+\tau_{f} \varepsilon I_{0}} .
$$

Substituting Eq. (A6) into Eq. (A4) results in Eq. (7).

\section{APPENDIX B}

\section{Derivation of Eq. (10)}

By using Eq. (A3) and changing the integration area, the integration in Eq. (9) becomes

$$
C_{1}=s \tau_{f} I_{0}\left(e^{-\varepsilon\left(c^{0}-c_{1}(a)\right)}-e^{-\varepsilon\left(c^{0}-c_{1}(b)\right)}\right) .
$$

Neglecting the ground-state depletion in the exponential term in Eq. (5) while preserving the inhomogeneous distribution effect, Eq. (5) becomes

$$
c_{1}(x)=\tau_{f} \varepsilon I_{0}\left(c^{0}-c_{1}(x)\right) e^{-\varepsilon c^{0} x} .
$$

Thus

$$
c_{1}(x)=\frac{\tau_{f} \varepsilon c^{0} I_{0} e^{-\varepsilon c^{0} x}}{1+\tau_{f} \varepsilon I_{0} e^{-\varepsilon c^{0} x}},
$$

by which $c_{1}(a)$ and $c_{1}(b)$ can be determined. Incorporating $c_{1}(a)$ and $c_{1}(b)$ into Eq. (B1) and with reference to Eq. (8) gives Eq. (10).

\section{APPENDIX C}

\section{Derivation of Eq. (11)}

According to scheme 1, the kinetics for the back ET reaction is expressed as

$$
\frac{d\left[D^{+\bullet}\right]}{d t}=k_{-e(g)}\left[D^{+\bullet} \cdot \bullet \cdot A^{-\bullet}\right],
$$

while the experimentally observed second order rate constant is defined as

$$
\frac{d\left[D^{+\bullet}\right]}{d t}=k_{\mathrm{obs}}^{b}\left[D^{+\bullet}\right]\left[A^{-\bullet}\right] .
$$

Under steady-state approximation, the steady-state concentration of $\left[D^{+} \cdot \bullet \cdot A^{-\bullet}\right]$ has the following expression:

$$
\left[D^{+} \bullet \bullet \cdot A^{-\bullet}\right]=\frac{k_{d}^{\prime}\left[D^{+\bullet}\right]\left[A^{-\bullet}\right]+k_{e}\left[D^{*} \cdot \bullet A\right]}{k_{-d}^{\prime}+k_{-e(g)}+k_{-e}} .
$$

Combining the three equations gives Eq. (11).

\section{APPENDIX D}

\section{Derivation of Eq. (14)}

The initial concentration of the escaped radical ions can be calculated from a slightly modified form of scheme 1 , and the result is expressed as ${ }^{19}$

$$
\left[D^{+\bullet}\right]_{0}=\left[A^{-\bullet}\right]_{0}=\frac{k_{q} k_{-d}^{\prime}[A]\left[D^{*}\right]_{0}}{\left(k_{q}[A]+k_{0}\right)\left(k_{-d}^{\prime}+k_{-e(g)}\right)},
$$

where $k_{q}$ is the Stern-Volmer quenching rate constant and $k_{0}$ is the radiative decay rate of the excited-triplet state. $D^{+\bullet}$ and $A^{-\cdot}$ would follow a second order decay with an intrinsic rate constant of $k_{\mathrm{ET}}^{b}$, assuming no diffusional pair $D^{*} \cdots A$ is formed. Then

$$
\left[D^{+\bullet}(t)\right]=\left[A^{-\bullet}(t)\right]=\frac{\left[D^{+\bullet}\right]_{0}}{\left(1+\left[D^{+\bullet}\right]_{0} k_{\mathrm{ET}}^{b} t\right)} .
$$

At the early stage of the decay phase, i.e., $\left[D^{+\bullet}\right]_{0} k_{\mathrm{ET}} t \ll 1$, $\left[D^{+\bullet}(t)\right]$ and $\left[A^{-\bullet}(t)\right]$ are proportional to $\left[D^{+\bullet}\right]_{0}$, which are in turn linearly correlated to $\left[D^{*}\right]_{0}$ by Eq. (D1). While at the steady state, the concentration of $D^{*} \bullet \bullet A$ has the following form:

$$
\left[D^{* \cdots A} \cdot(t)\right]=\frac{k_{d}[A]\left[D^{*}(t)\right]}{k_{-d}} .
$$

At the initial stage, $D^{*}$ would decay in a monoexponential way; when a steady state is achieved, the concentration would remain constant. If $t_{s}$ is the time taken for buildup of the steady state $\left(t_{s}\right.$ is fixed for a monoexponential decay process), then under the steady state assumption,

$$
\left[D^{*} \cdots A(t)\right]=\frac{k_{d}[A]\left[D^{*}\right]_{0} e^{-k_{q} t_{s}}}{k_{-d}} .
$$

Substituting Eqs. (D1), (D2), and (D4) into Eq. (12), and setting the time domain such that $\left[D^{+\bullet}\right]_{0} k_{\mathrm{ET}} t \ll 1$, Eq. (12) becomes

$$
\begin{aligned}
k_{\mathrm{obs}}^{b} \approx & \frac{k_{-e(g)} k_{d}^{\prime}}{k_{-d}^{\prime}}+\frac{k_{-e(g)} k_{e}}{k_{-d}^{\prime}} \\
& \times\left\{\frac{\left(k_{q}[A]+k_{0}\right)\left(k_{-d}^{\prime}+k_{-e(g)}\right)}{k_{q} k_{-d}^{\prime}}\right\}^{2} \frac{k_{d} e^{-k_{q} t_{s}}}{k_{-d}[A]\left[D^{*}\right]_{0}} .
\end{aligned}
$$

Equation (D5) predicts that $k_{\mathrm{obs}}^{b}$ is linearly proportional to the reciprocal of $\left[D^{*}\right]_{0}$, while $\left[D^{*}\right]_{0}$ is expressed by Eq. (8). Substituting Eq. (13) into Eq. (D5) gives Eq. (14). 
${ }^{1}$ R. A. Marcus, J. Chem. Phys. 24, 966 (1956).

${ }^{2}$ R. A. Marcus and N. Sutin, Biochim. Biophys. Acta 811, 265 (1985).

${ }^{3}$ D. Rehm and A. Weller, Isr. J. Chem. 8, 259 (1970).

${ }^{4}$ C. Creutz and N. Sutin, J. Am. Chem. Soc. 99, 241 (1977).

${ }^{5}$ J. K. Nagle, W. J. Dressick, and T. J. Meyer, J. Am. Chem. Soc. 101, 3993 (1979).

${ }^{6}$ J. R. Miller, L. T. Calcaterra, and G. L. Closs, J. Am. Chem. Soc. 106, 3047 (1984).

${ }^{7}$ J. R. Miller, J. V. Beitz, and R. K. Huddleston, J. Am. Chem. Soc. 106, 5057 (1984).

${ }^{8}$ G. L. Closs, L. T. Calcaterra, N. J. Green, K. W. Penfield, and J. R. Miller, J. Phys. Chem. 90, 3673 (1986)

${ }^{9}$ G. L. Closs and J. R. Miller, Science 240, 440 (1988).

${ }^{10}$ G. Mclendon and R. J. Miller, J. Am. Chem. Soc. 107, 7811 (1985).

${ }^{11}$ M. R. Wasielewski, M. P. Niemczyk, W. A. Svec, and E. B. Pewitt, J. Am. Chem. Soc. 107, 1080 (1985).

${ }^{12}$ S. Nishitani, N. Kurata, Y. Sakata, S. Misumi, A. Karen, T. Okada, and T. Mataga, J. Am. Chem. Soc. 105, 7771 (1983).

${ }^{13}$ L. S. Fox, M. Kozik, J. R. Winkler, and H. B. Gray, Science 247, 1069 (1990).

${ }^{14}$ R. S. Farid, I.-J Chang, J. R. Winkler, and H. B. Gray, J. Phys. Chem. 98, 5176 (1994)

${ }^{15}$ I. R. Gould, D. Ege, S. L. Mattes, and S. Farid, J. Am. Chem. Soc. 109, 3794 (1987).

${ }^{16}$ I. R. Gould, J. E. Moser, B. Armitage, S. Farid, J. L. Goodman, and M. S. Herman, J. Am. Chem. Soc. 111, 1917 (1989).

${ }^{17}$ I. R. Gould, J. E. Moser, D. Ege, and S. Farid, J. Am. Chem. Soc. 110, 199 (1988).

${ }^{18}$ I. R. Gould and S. Farid, J. Am. Chem. Soc. 110, 1991 (1988).

${ }^{19}$ G. Grampp and G. Hetz, Ber. Bunsenges. Phys. Chem. 96, 198 (1992).

${ }^{20}$ T. M. McCleskey, J. R. Winkler, and H. B. Gray, J. Am. Chem. Soc. 114, 6935 (1992).

${ }^{21}$ K. C. Cho, C. M. Che, K. M. Ng, and C. L. Choy, J. Phys. Chem. 91, 3690 (1987).

${ }^{22}$ T. Ohno, A. Yoshimura, and N. Mataga, J. Phys. Chem. 90, 3295 (1986).

${ }^{23}$ C. Turro, J. M. Zaleski, Y. M. Karabatsos, and D. G. Nocera, J. Am. Chem. Soc. 118, 6060 (1996).

${ }^{24}$ R. M. Noyes, J. Am. Chem. Soc. 77, 2042 (1955).

${ }^{25}$ R. M. Noyes, J. Am. Chem. Soc. 78, 5468 (1956).

${ }^{26}$ C. M. Che, H. L. Kwong, C. K. Poon, and V. W. W. Yam, J. Chem. Soc. Dalton Trans. 3215 (1990).
${ }^{27}$ L. A. Kelly and M. A. J. Rodgers, J. Phys. Chem. 99, 13132 (1995).

${ }^{28}$ R. Castro, M. L. Duran, J. A. Garcia-Vazquez, J. Romero, A. Sousa, E. E. Castellano, and J. Zukerman-Schpector, J. Chem. Soc. Dalton Trans. 2559 (1992).

${ }^{29}$ S. Seth, A. K. Das, and T. C. W. Mak, Acta Crystallogr., Sect. C: Cryst. Struct. Commun. 51, 2529 (1995).

${ }^{30}$ U. Lachish, A. Shafferman, and G. Stein, J. Chem. Phys. 64, 4205 (1976).

${ }^{31}$ E. Pitts, G. C. Terry, and F. W. Willets, Trans. Faraday. Soc. II 62, 2858 (1966)

${ }^{32}$ H. S. Pilloff and A. C. Albrecht, J. Chem. Phys. 49, 4891 (1968).

${ }^{33}$ N. I. Avdievich, A. S. Jeevarajan, and M. D. E. Forbes, J. Phys. Chem. 100, 5334 (1996).

${ }^{34}$ F. Scandola, V. Balzani, and G. B. Schuster, J. Am. Chem. Soc. 103, 2519 (1981).

${ }^{35}$ V. Balzani and A. Juries, Coord. Chem. Rev. 84, 85 (1988).

${ }^{36}$ G. N. Lewis and M. Kasha, J. Am. Chem. Soc. 66, 2100 (1944).

${ }^{37}$ K. D. Cadogan and A. C. Albrecht, J. Phys. Chem. 72, 929 (1968).

${ }^{38}$ S. Kitagawa, M. Munakata, H. Shimono, S. Matsuyama, and H. Masuda, J. Chem. Soc. Dalton Trans. 2105 (1990).

${ }^{39}$ C. M. Che, H. L. Kwong, C. K. Poon, V. W.-W. Yam, and K. C. Cho, J. Chem. Soc. Chem. Commun. 885 (1989).

${ }^{40}$ C. King, J.-C. Wang, Md. N. I. Khan, and J. P. Fackler, Jr., Inorg. Chem. 28, 2145 (1989)

${ }^{41}$ J. N. Demas and G. A. Crosby, J. Phys. Chem. 75, 991 (1971).

${ }^{42}$ J. P. Simons, Photochemistry and Spectroscopy (Wiley-Interscience, London, 1971).

${ }^{43}$ R. Potashnik, M. Ottolenghi, and R. Bensasson, J. Phys. Chem. 73, 1912 (1969).

${ }^{44}$ K. C. Cho and C. M. Che, Chem. Phys. Lett. 124, 313 (1986).

${ }^{45}$ B. S. Brunschwig and N. Sutin, Comments Inorg. Chem. 6, 209 (1987)

${ }^{46}$ J. Ulstrup and J. Jortner, J. Chem. Phys. 63, 4358 (1975).

${ }^{47}$ D. N. Beratan, J. N. Onuchic, J. R. Winkler, and H. B. Gray, Science 258, 1740 (1992)

${ }^{48}$ T. Watanabe and K. Honda, J. Phys. Chem. 86, 2617 (1982).

${ }^{49}$ J. Hermolin, M. Levin, and E. M. Kosower, J. Am. Chem. Soc. 103, 4808 (1981).

${ }^{50}$ J. A. Farrington, M. Ebert, and E. J. Land, J. Chem. Soc., Faraday Trans. 1665 (1978).

${ }^{51}$ J. Hermolin, M. Levin, and E. M. Kosower, J. Am. Chem. Soc. 103, 4808 (1981). 